\title{
Surgical management of congenital spinal lesions associated with abnormalities of the cranio-spinal junction ${ }^{1}$
}

\author{
W. BRADFORD DELONG AND RICHARD C. SCHNEIDER \\ From the Department of Surgery, Section of Neurosurgery, University of Michigan \\ Medical Center, Ann Arbor, Michigan, U.S.A.
}

The possibility of inducing a fatal cerebellomedullary pressure cone as the result of lumbar puncture in a patient with increased intracranial pressure has long been recognized by physicians and surgeons. It follows rather obviously that the release of cerebrospinal fluid during spinal surgery in a patient with eleyated intracranial pressure could also cause a similar fatal outcome, but this point has not been adequately stressed in the literature.

Pertinent to this, however, is the mention by Laurence (1964) of three patients, aged 3, 5, and 6 years, with apparently arrested hydrocephalus, each of whom died shortly after the repair of a myelomeningocele. Doran and Guthkelch (1961), also, discuss a 6-year-old girl who required medullary decompression 48 hours after repair of a myelomeningocele. In order to emphasize further the risks involved when surgery is directed at congenital spinal lesions in patients past infancy, we wish to describe two patients, both of whom had lower spinal pathology associated with abnormalities of the cranio-spinal junction, and we wish to state our current conclusions concerning the surgical management of such patients.

\section{CASE REPORTS}

CASE 1 J.M. was a 4-year-old boy admitted to the University Medical Center on 8 April 1951. His motor development had been somewhat retarded, and he had not started walking until the age of 2 . Then, within the next year, his lower extremities had become weak and spastic, and he had reverted to crawling. Examination disclosed a large head, spastic paraplegia, bilateral extensor plantar reflexes, and a palpably open lumbosacral spinal canal. No sensory deficit or cranial nerve palsies could be demonstrated.

Skull radiographs demonstrated a large foramen magnum, a small posterior fossa, and basilar impression. Spine films showed a widened interpeduncular distance in the lumbosacral region, and myelography a markedly

${ }^{1} A$ part of this study was supported by the Begole-Brownell Fund. abnormal lumbosacral dural sac with multiple pouches of dura at several levels and a block to the superior flow of contrast material at the first lumbar vertebra.

The pathology in the lumbosacral area provided the most likely explanation for the paraplegia, and in an effort to halt the progression of this deficit, a laminectomy was undertaken. The spinal canal was exposed from the twelfth thoracic through the first sacral levels, and the posterior osseous and ligamentous structures were found to be markedly abnormal, with fibrous bands lying transversely across the dura at each interspace. Intradural exploration disclosed the spinal cord to be matted against the dura anteriorly and to extend into the sacral region. The cord was engulfed in connective tissue at the level of the interspace between the third and fourth lumbar vertebrae and a moderate amount of intradural fat was present at the level of the fourth and fifth lumbar vertebrae (Fig. 1). There was a thick fibrous band around the cord at the level of the first lumbar vertebra, and this was dissected free of the cord. When a similar band at the twelfth thoracic vertebra was incised, spinal fluid gushed down from above, and a catheter passed superiorly in the subarachnoid space proved no significant block to be present superior to this vertebra. At this point in the operation the child's blood pressure suddenly dropped, the pulse became weak and rapid, and his condition deteriorated rapidly. He expired in spite of resuscitative attempts.

The post-mortem examination confirmed the small posterior fossa and the basilar impression seen on the radiographs. Marked dilatation of the ventricles was present, including the fourth. The pathologist described 'a well-developed cerebellar pressure cone', and there was congestion of the capillaries in the upper cervical spinal cord segments. There were multiple cysts and small vascular anomalies of the meninges at several levels.

Apparently, the basilar impression alone accounted for the hydrocephalus, since there was no evidence of the Arnold-Chiari malformation or other pathology in a location which would have blocked the circulation of cerebrospinal fluid. In any case, we believe that the release of spinal fluid at the thoraco-lumbar junction caused sudden and devastating 


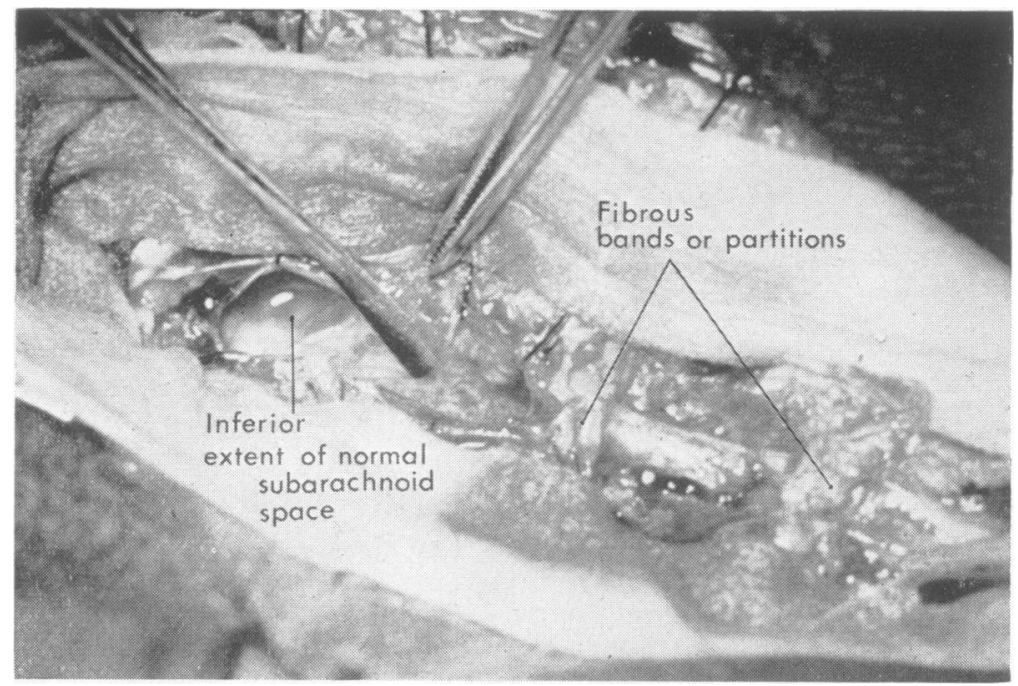

FIG. 1. Operative photograph of case 1 demonstrating the fibrous bands surrounding the spinal cord in the lumbar region. Note that the subarachnoid space was minimally developed inferior to the thoraco-lumbar junction.

medullary compression as a result of the increased pressure differential across the foramen magnum. The capillary congestion in the cervical spinal cord may have been secondary to an acute process occurring in that region, and the gross picture was that of a typical cerebellar pressure cone.

CASE 2 J.H. was 14 years old at the time of her admission to the University Medical Center on 13 October 1958. She had a lumbosacral myelomeningocele surgically closed shortly after birth, then developed hydrocephalus which had spontaneously arrested within a few weeks. By the age of 5 years, she was able to walk. Because of severe urinary tract disease, an ileal bladder had been constructed when she was 12 years old. She had always noted anaesthesia of the inferior portion of the left lower extremity and saddle area, but in July 1958 the sensory deficit began to increase and soon she developed weakness of both lower extremities, which progressed so that at the time of admission she could not walk.

Examination revealed a head circumference of $22 \frac{1}{2}$ in., which represented no change from the circumference reported in 1955. There was nystagmus on lateral gaze to either side, but otherwise the cranial nerves were intact. She had marked weakness of all muscle groups in both lower extremities with bilateral patellar clonus and a right extensor plantar reflex. Strength in the upper extremities was also moderately diminished. Sensation to pin prick was markedly impaired below the ninth thoracic dermatomes bilaterally.

Lumbosacral spine radiographs demonstrated a marked spina bifida, and a myelogram, performed between the twelfth thoracic and first lumbar spinous processes, superior to the region of the repaired myelomeningocele, demonstrated a block to the superior flow of contrast material at the tenth thoracic level. A ventriculogram disclosed marked dilatation of the lateral and third ventricles, and the fourth ventricle could not be तु visualized. The posterior fossa was small.

It was felt that the progressive weakness of the lowes extremities was a result of lower thoracic spinal pathologx $\overrightarrow{0}$ and a laminectomy was carried out, the laminae of the 응 tenth thoracic through the second lumbar vertebrae being removed. As this exposure was being accomplished, how ever, her blood pressure climbed from $120 / 94 \mathrm{~mm}$. Hg to $160 / 130 \mathrm{~mm}$. $\mathrm{Hg}$ and her condition began to deteriorate The suboccipital region had been prepared before draping, and as this region was being quickly exposed, the ventricle was tapped. A suboccipital craniectomy was' o performed and the laminae of the first and second cervical vertebrae were removed. A dense extradural fibrous band at the level of the foramen magnum was incised and the dura opened. Dense adhesions bound the medulla to the cerebellum and surrounding dura, and the first and second cervical nerve roots were directed superiorly. This was felt to be a typical Arnold-Chiari malformation. Lysis of the adhesions surrounding the medulla was limited by severe bleeding occurring during the dissection, but adhesions in the region of the foramen of Magendie were freed so that indigo carmine instilled into the lateral ventricle flowed from the fourth ventricle into the operative field. The dura overlying the foramen magnum was left open. Attention was again turned to the thoracolumbar junction, and intradural exploration demonstrated an atrophic spinal cord adherent to the right side of the dural sac at the level of the twelfth thoracic vertebra. The cord was freed as well as possible, and both incisions were closed.

Unfortunately, her weakness and spasticity failed to respond to the surgery, and in March 1959, during a subsequent admission, ventricular puncture disclosed elevated pressure. A ventriculo-atrial shunt was performed, and following this procedure, she improved to some degree, eventually walking with crutches and braces. 
This girl developed medullary compression either as a result of surgical manipulation in the thoracolumbar region or from increased intracranial pressure as a result of the general anaesthetic (or perhaps from a combination of both factors), but a ventricular tap and decompression of the brain-stem at the foramen magnum countered the deterioration. The Arnold-Chiari malformation was evidently responsible for the pre-existing hydrocephalus, but the circulation of cerebrospinal fluid did not seem to be improved by the dissection of the adhesions in the posterior fossa, since a ventriculo-atrial shunt was later required to reduce the increased ventricular pressure.

\section{DISCUSSION}

Though the first case represents a somewhat different situation, the most common example of associated lumbo-sacral and cranio-spinal pathology is represented by a myelomeningocele associated with the Arnold-Chiari malformation. The close relationship of the two lesions was described by Schwalbe and Gredig (1907), was documented in English by Russell and Donald (1935), and has been recently reemphasized by Cameron (1957), who found 22 cases of the typical malformation in 26 cases of spina bifida cystica with atypical variants in two of the remaining cases. The high incidence of hydrocephalus in patients presenting with myelomeningoceles has been emphasized by Lorber (1961), who points out that among paraplegic infants with lumbar lesions there is a $96 \%$ incidence of dilated ventricles.

Repair of a myelomeningocele is precarious in the infant with markedly increased intracranial pressure (Ingraham and Matson, 1954), and, we feel, in the patient whose fontanelles have closed. The danger in the latter instance is, of course, the presence of occult hydrocephalus. The release of fluid inferiorly can cause a significant increase in the pressure differential between the closed intracranial cavity and the spinal canal. This may result in either immediate impaction of the medulla in the upper cervical region or further impairment in cerebrospinal fluid circulation with subsequent increased intracranial pressure and resultant medullary impaction.

We believe that immediate impaction of the medulla occurred in case 1 as a result of the release of spinal fluid, and in case 2 as a result either of the decompressive effect of the lumbar laminectomy itself or of increased intracranial pressure from the general anaesthetic. In the first instance, death rapidly followed, but in case 2 , a rapidly performed ventricular puncture followed by medullary decompression reversed the progression of events. As
Doran and Guthkelch (1961) have pointed out, in order to relieve neurological compromise secondary to the Arnold-Chiari malformation, surgical decompression of the medulla in the foramen magnum and upper cervical region may be necessary even after elevated intracranial pressure has been relieved by a shunting procedure. Conversely, a direct approach to the malformation itself may not relieve hydrocephalus and a ventriculo-atrial shunt may be necessary subsequently, as was demonstrated in the second patient described above.

Medullary compression seems to be avoided if the myelomeningocele is repaired during infancy, provided the intracranial pressure is not markedly elevated before the repair. The dangers of lesser degrees of intracranial pressure or of subclinical hydrocephalus are offset by the decompression afforded by the open fontanelles. Further protection may also be afforded by the fact that the hydrocephalus in a certain percentage of these infants is of the communicating type (Ingraham and Matson, 1954). It is possible that as the patient grows older the adhesions in the region of the Arnold-Chiari malformation become more dense, converting the hydrocephalus to the non-communicating type, thus increasing the risks of medullary compromise during myelomeningocele repair as spinal fluid is released. This postulate does not apply, of course, to those patients having non-communicating hydrocephalus in the first place, and, as Lichtenstein $(1942,1959)$ has pointed out, a number of patients with the Arnold-Chiari malformation have a malformation or stenosis of the aqueduct of Sylvius.

No matter how old the patient in whom the repair of a myelomeningocele is contemplated, we feel that the presence of an associated Arnold-Chiari malformation should be assumed, and in the patient with closed fontanelles the degree of ventricular dilatation and intracranial pressure should be assessed before the repair by means of a ventricular puncture and a ventriculogram. In the presence of dilated ventricles, a ventricular pressure within the currently accepted normal range of values (50 to $180 \mathrm{~mm}$. $\mathrm{H}_{2} \mathrm{O}$, DeJong, 1958) does not necessarily indicate normal intracranial hydrodynamics, as Adams, Fisher, Hakim, Ojeman, and Sweet (1965) have recently emphasized.

We now feel that the patients with ventricular dilatation and closed fontanelles, even though they may have 'normal' ventricular pressure, should be protected with a ventriculostomy performed just before the repair of the spinal lesion, with ventricular drainage maintained into the postoperative period. If increased intracranial pressure is present preoperatively, or develops postoperatively, then a ventriculoatrial shunt should be performed as soon 
as the danger of infection in the spinal wound has passed. We feel that the insertion of a permanent shunt should be deferred, if possible, until after the spinal surgery has been performed in order to avoid compromising the shunting device either by infection, if this should unfortunately develop in the spinal wound, or by blood and air in the ventricular and subarachnoid compartments if a suboccipital operation should become necessary.

The patient with a myelomeningocele who shows normal ventricular size and pressure on preoperative assessment will be rare, and in this situation we would be inclined to proceed with the repair of the spinal lesion, with adequate provision having been made for the expeditious institution of ventricular drainage if the patient's condition deteriorates.

In any of the above situations, even though ventricular drainage has been instituted, it may become necessary to perform medullary decompression if the patient shows signs of medullary compromise, either at the time of the spinal procedure or after operation. The suboccipital and cervical regions should be prepared and draped along with the region of the spinal lesion itself, to allow for rapid suboccipital craniectomy and cervical laminectomy if this should become necessary during the spinal operation. Postoperatively, the possible necessity for such intervention should be kept in mind.

The first case described above emphasizes that whenever surgical intervention after infancy is considered for congenital lesions of the spinal region, then a reasonable search should be made for associated intracranial or craniospinal lesions, and further preliminary studies performed as indicated. The possible necessity for complementary decompressive procedures before or at the time of the spinal operation should be recognized.

\section{SUMMARY}

We have described two patients, both of whom had a lumbar lesion associated with pathology at the craniospinal junction without significant cranial nerve signs. Both also had hydrocephalus without signs of increased intracranial pressure. The death of one and the crisis occurring in the other at the time of spinal surgery illustrate an occult risk involved in dealing with patients having congenital spinal lesions, many of whom may also have unrecognized hydrocephalus.

We feel that the repair of such lesions during infancy, as is usually done in the case of myelomeningoceles, largely avoids this risk, and that if repair must be done after the fontanelles have closed, then extreme caution must be used to avoid medullary. compromise during or shortly after the repair. We have suggested a programme of surgical management designed to minimize the risk of medullary compression.

\section{REFERENCES}

Adams, R. D., Fisher, C. M., Hakim, S., Ojemann, R. G., and Sweet, W. H. (1965). Symptomatic occult hydrocephalus with 'normal' cerebrospinal fluid pressure: A treatable syndrome. New Eng. J. Med., 273, 117-126.

Cameron, A. H. (1957). The Arnold-Chiari and other neuro-anatomical malformations associated with spina bifida. J. Path. Bact., 73, $195-211$.

DeJong, R. N. (1958). The Neurologic Examination, 2nd ed., p. 975 Hoeber, New York.

Doran, P. A., and Guthkelch, A. N. (1961). Studies in spina bifida cystica. I. General survey and reassessment of the problem J. Neurol. Neurosurg. Psychiat., 24, 331-345.

Ingraham, F. D., and Matson, D. D. (1954). Neurosurgery of Infanc and Childhood, pp. 19-48. Thomas, Springfield, Illinois.

Laurence, K. M. (1964). The natural history of spina bifida cystica $\delta$ Detailed analysis of 407 cases. Arch. Dis. Child., 39, 41-57.

Lichtenstein, B. W. (1942). Distant neuroanatomic complications of spina bifida (spinal dysraphism): Hydrocephalus, Arnold $\overline{-}$ Chiari deformity, stenosis of the aqueduct of Sylvius, etc. pathogenesis and pathology. Arch. Neurol. Psychiat. (Chic.) 47, $195-214$.

(1959). Atresia and stenosis of the aqueduct of Sylvius, with comments on the Arnold-Chiari complex. J. Neuropath. exp. Neurol., 18, 3-21.

Lorber, J. (1961). Systematic ventriculographic studies in infants born with meningomyelocele and encephalocele: the incidence and development of hydrocephalus. Arch. Dis. Child., 36, 381-389.

Russell, D. S., and Donald, C. (1935). The mechanism of internal hydrocephalus in spina bifida. Brain, 58, 203-215.

Schwalbe, E., and Gredig, M. (1907). Über Entwicklungsstörungen des Kleinhirns, Hirnstamms und Halsmarks bei Spina bifida (Arnold'sche und Chiari'sche Missbildung). Beitr. path. Anat. 40, 132-194. 